\title{
To Study the Antimicrobial Properties of Honey on Common Microorganisms
}

\author{
P.S. Aswin and P. Neelusree* \\ Department of Microbiology, Saveetha Medical College and Hospital, India \\ *Corresponding author
}

\section{A B S T R A C T}

\begin{abstract}
Honey is defined as a natural sweet substance which is produced from the floral nectar by honeybees. Honey is well-known for its antimicrobial activities and it has been reported to have antibacterial effect to about 60 species of bacteria and antifungal properties as well. Honey has been used to inhibit these bacteria as well as to prevent and treat skin and other infections. Honey as a tropical antibacterial agent for treatment of infected wounds. The natural ingredients of honey show different activities against various microorganisms. Honey has an increasing effect on the levels of anti-oxidants, iron and rare elements in blood. The antimicrobial property is also based on UMF of that honey taken showed that honey had a more pronounced inhibitory effect $(85.7 \%)$ on Gram negative bacteria (Pseudomonas aeruginosa, Enterobacter spp., Klebsiella) in comparison to commonly used antimicrobial agents. To analyse the establishment activity of different honey (Coorg honey, Kashmir Herbal honey, Natural Kashmir honey, Dabur honey and Manuka honey) on various organisms like Acinetobacter, Coagulase Negative Staphylococcus (CONS), Methicillin resistant Coagulase Negative Staphylococcus (MRCONS), Methicillin Sensitive Staphylococcus aureus (MSSA), Methicillin resistant Staphylococcus aureus (MRSA), Enterococcus, Klebsiella, E. coli, ATCC Staphylococcus aureus, Pseudomonas With various concentration $(10 \%, 20 \%, 50 \%)$. Among all the brands of honey tested Manuka honey was effective against ever against all the organisms tested at $50 \%$ concentration. Similarly Dabur Honey was effective against all organisms at $50 \%$. In conclusion, though Manuka honey is the best for treating microorganisms, it is not affordable to low socioeconomic people, because of its high cost. Still Dabur Honey can be an alternative topical agent to treat burns wound infections which are cost effective. Honey exhibits antimicrobial property against gram negative and few gram positive organisms.
\end{abstract}

\section{Keywords}

Coorg honey, Kashmir Herbal honey, Natural Kashmir honey, Dabur honey and Manuka honey

Article Info

Accepted: 04 August 2019 Available Online: 10 September 2019

\section{Introduction}

Honey is defined as a natural sweet substance which is produced from the floral nectar by honeybees. Honey is well-known for its antimicrobial activities and it has been reported to have antibacterial effect to about 60 species of bacteria and antifungal properties as well [1]. Honey has been used to inhibit these bacteria as well as to prevent and 
treat skin and other infections [2]. Honey as a tropical antibacterial agent for treatment of infected wounds. [3]. The natural ingredients of honey show different activities against various microorganisms. Honey has an increasing effect on the levels of anti-oxidants, iron and rare elements in blood [4]. The antimicrobial property is also based on UMF of that honey taken [5] showed that honey had a more pronounced inhibitory effect $(85.7 \%)$ on Gram negative bacteria (Pseudomonas aeruginosa, Enterobacter spp., Klebsiella) in comparison to commonly used antimicrobial agents. The use of honey as a drug for the treatment of disease dates back to 2100-2000 BC. For instance, pale honey was described by Aristotle (384-322 BC) as being "good for sore eyes and wounds" [6]. A possible reason behind its activity relies on its ability to generate hydrogen peroxide by the beederived enzyme glucose oxidase [7].

\section{Materials and Methods}

The present prospective study was carried out in the department of Microbiology, Saveetha Medical college. This study was done to analyse the establishment activity of different honey on various organisms like Acinetobacter, Coagulase Negative Staphylococcus (CONS), Methicillin resistant Coagulase Negative Staphylococcus (MRCONS), Methicillin Sensitive Staphylococcus aureus (MSSA), Methicillin resistant Staphylococcus aureus (MRSA), Enterococcus, Klebsiella, E. coli, ATCC Staphylococcus aureus, Pseudomonas. Each of these organisms was treated with Five different honey (Coorg honey, Kashmir Herbal honey, Natural Kashmir honey, Dabur honey and Manuka honey) with various concentration of $50 \%, 20 \%$ and $10 \%$.

\section{Preparation of honey solution}

To a $10 \mathrm{ml}$ of sterilised nutrient broth I added $10 \mathrm{ml}$ of honey to prepare the concentration of
$50 \%$. To a $16 \mathrm{ml}$ of sterilised nutrient broth I added $4 \mathrm{ml}$ of honey to prepare a concentration of $20 \%$. To a $18 \mathrm{ml}$ of sterilised nutrient broth I added $2 \mathrm{ml}$ of honey to prepare a concentration of $10 \%$. A loopful of each microorganism (Acinetobacter, Coagulase Negative Staphylococcus (CONS),Methicillin resistant Coagulase Negative Staphylococcus (MRCONS), Methicillin Sensitive Staphylococcus aureus (MSSA),Methicillin resistant Staphylococcus aureus (MRSA), Enterococcus, Klebsiella, E. coli, ATCC Staphylococcus aureus, Pseudomonas) is mixed to each concentration $10 \% 20 \%$ and $50 \%$ concentration of different types of honey (Coorg honey, Kashmir Herbal honey, Natural Kashmir honey, Dabur honey and Manuka honey) respectively and is incubated overnight. After overnight incubation, it is streaked onto Muller Hinton Agar and incubated at $37^{*} \mathrm{c}$ for 24 hours. After 24 hours incubation the plates were observed for the growth on each concentration.

\section{Results and Discussion}

Prospective study carried out in Department of Microbiology, Saveetha Medical College to analyse the antibacterial activity of various brands of honey (Coorg honey, Kashmir Herbal honey, Natural Kashmir honey, Dabur honey and Manuka honey) on various organisms [Acinetobacter, Coagulase Negative Staphylococcus (CONS),Methicillin resistant Coagulase Negative Staphylococcus (MRCONS), Methicillin Sensitive Staphylococcus aureus (MSSA), Methicillin resistant Staphylococcus aureus (MRSA), Enterococcus, Klebsiella, E. coli, ATCC Staphylococcus aureus, Pseudomonas] at various concentrations $10 \%, 20 \%$ and $50 \%$.

\section{Coorg Honey}

Antibacterial activity against various organisms [Acinetobacter, Coagulase Negative Staphylococcus (CONS), Methicillin 
resistant Coagulase Negative Staphylococcus (MRCONS), Methicillin Sensitive Staphylococcus aureus (MSSA), Methicillin resistant Staphylococcus aureus (MRSA), Enterococcus, Klebsiella, E. coli, ATCC Staphylococcus aureus, Pseudomonas] analysed at 10\%, 20\% and 50\% concentrations. It showed $50 \%$ concentration was effective in killing Enterococcus and CONS. None other $10 \%$ nor $20 \%$ concentration was effective against other organisms tested (As shown in Fig. 1).

\section{Kashmir herbal honey}

Antibacterial activity against various organisms [Acinetobacter, Coagulase Negative Staphylococcus (CONS),Methicillin resistant Coagulase Negative Staphylococcus (MRCONS), Methicillin Sensitive Staphylococcus aureus (MSSA), Methicillin resistant Staphylococcus aureus (MRSA), Enterococcus, Klebsiella, E. coli, ATCC Staphylococcus aureus, Pseudomonas] analysed at 10\%, 20\% and 50\% concentrations. It showed 50\% concentration was effective against MRSA and MSSA; and $20 \%$ concentration was found to be effective against CONS. This honey was found ineffective against other organisms (As shown in Fig. 2).

\section{Natural Kashmir honey}

Antibacterial activity against various organisms [Acinetobacter, Coagulase Negative Staphylococcus (CONS), Methicillin resistant Coagulase Negative Staphylococcus (MRCONS), Methicillin Sensitive Staphylococcus aureus (MSSA), Methicillin resistant Staphylococcus aureus (MRSA), Enterococcus, Klebsiella, E. coli, ATCC Staphylococcus aureus, Pseudomonas] analysed at 10\%, 20\% and 50\% concentrations. It showed 50\% concentration was effective against CONS. None other concentration was effective against other organisms (Fig. 3).

\section{Dabur honey}

Antibacterial activity against various organisms [Acinetobacter, Coagulase Negative Staphylococcus (CONS),Methicillin resistant Coagulase Negative Staphylococcus (MRCONS), Methicillin Sensitive Staphylococcus aureus (MSSA), Methicillin resistant Staphylococcus aureus (MRSA), Enterococcus, Klebsiella, E. coli, ATCC Staphylococcus aureus Staphylococcus (MRCONS) Methicillin Sensitive Staphylococcus aureus (MSSA), Methicillin resistant Staphylococcus aureus (MRSA), Enterococcus, Klebsiella, E. coli, ATCC Staphylococcus aureus, Pseudomonas] (As shown is Fig. 4).

\section{Manuka honey}

Antibacterial activity against various organisms [Acinetobacter, Coagulase Negative Staphylococcus (CONS), Methicillin resistant Coagulase Negative Staphylococcus (MRCONS), Methicillin Sensitive Staphylococcus aureus (MSSA), Methicillin resistant Staphylococcus aureus (MRSA), Enterococcus, Klebsiella, E. coli, ATCC Staphylococcus aureus, Pseudomonas] analysed at 10\%, 20\% and 50\% concentrations.

It showed 50\% was effective against all microorganisms [Acinetobacter, Coagulase Negative Staphylococcus (CONS), Methicillin resistant Coagulase Negative Staphylococcus (MRCONS), Methicillin Sensitive Staphylococcus aureus (MSSA), Methicillin resistant Staphylococcus aureus (MRSA), Enterococcus, Klebsiella, E. coli, ATCC Staphylococcus aureus, Pseudomonas]; and $20 \%$ was effective against Acinetobacter, Coagulase Negative Staphylococcus (CONS), 
Methicillin resistant Coagulase Negative Staphylococcus (MRCONS), Methicillin Sensitive, Staphylococcus aureus (MSSA),Methicillin resistant Staphylococcus aureus (MRSA), Enterococcus, Klebsiella, E. coli.; and $10 \%$ was effective against Acinetobacter. One of the commonest multi drug resistant (MDR) organism in hospital setting (Acinetobacter) is killed by $10 \%, 20 \%$ and $50 \%$ concentration of Manuka Honey (As shown in Fig. 5).

Prospective study carried out in Department of Microbiology, Saveetha Medical College to analyse the antibacterial activity of various brands of honey (Coorg honey, Kashmir Herbal honey, Natural Kashmir honey, Dabur honey and Manuka honey) on various organisms [Acinetobacter, Coagulase Negative Staphylococcus (CONS), Methicillin resistant Coagulase Negative Staphylococcus (MRCONS), Methicillin Sensitive Staphylococcus aureus (MSSA), Methicillin resistant Staphylococcus aureus (MRSA), Enterococcus, Klebsiella, E. coli, ATCC Staphylococcus aureus, Pseudomonas] at various concentrations $10 \%, 20 \%$ and $50 \%$.

The antimicrobial properties of honey have been known to humans for centuries [8]. Honey was used to treat infected wounds as long ago as 2000 years before bacteria were discovered to be the cause of infection. In c.50 $\mathrm{AD}$, Dioscorides described honey as being "good for all rotten and hollow ulcers". Honey has been reported to have an inhibitory effect to around 60 species of bacteria including aerobes and anaerobes, gram-positives and gram-negatives [9].

An antifungal action has also been observed for some yeasts and species of Aspergillus and Penicillium, as well as all the common dermatophytes.

The current prevalence of antibiotic-resistant microbial species has led to a re-evaluation of the therapeutic use of ancient remedies, including honey [10]. Aristotle (384-322 BC), when discussing different honeys, referred to pale honey as being "good as a salve for sore eyes and wounds" Natural honey consists mainly of carbohydrates (about 82\%), water and other minor components. Those minor ingredients include: proteins, minerals, phytochemicals and antioxidants.

It has been reported that those minor ingredients are the ones that are responsible for medical and biological activities of honey in the treatment of infections, burns, wounds and ulcers. The honey sugars are mostly fructose $(38.2 \%)$ and glucose $(31.2 \%)$, sucrose concentration ranges between $(0.7 \%$ and $1 \%)$, disaccharides (approximately 9\%) some trisaccharides and higher saccharides [12].

Different types of honey possess different efficacies and mechanisms against the same type of bacteria, with Manuka Honey showing the best performance. This is in accordance with what has been reported by other authors who used honey as an agent to inhibit biofilm formation by $S$. aureus [13].

Manuka Honey contained the highest amount of total phenolic compounds such as methyl syringate which provides this honey with its ability to scavenge potent superoxide free radicals and, thus, exerts its antibacterial activity [14].

In our study antibacterial activity of Manuka honey was tested against various orgnisms [Acinetobacter, Coagulase Negative Staphylococcus (CONS),Methicillin resistant Coagulase Negative Staphylococcus (MRCONS), Methicillin Sensitive Staphylococcus aureus (MSSA), Methicillin resistant Staphylococcus aureus (MRSA), Enterococcus, Klebsiella, E. coli, ATCC Staphylococcus aureus, Pseudomonas] analysed at 10\%, 20\% and 50\% 
concentrations. It showed $50 \%$ was effective against all microorganisms [Acinetobacter, Coagulase Negative Staphylococcus (CONS), Methicillin resistant Coagulase Negative Staphylococcus (MRCONS), Methicillin Sensitive Staphylococcus aureus (MSSA), Methicillin resistant Staphylococcus aureus (MRSA), Enterococcus, Klebsiella, E. coli, ATCC Staphylococcus aureus, Pseudomonas]; and $20 \%$ was effective against Acinetobacter, Coagulase Negative Staphylococcus (CONS), Methicillin resistant Coagulase Negative Staphylococcus (MRCONS), Methicillin Sensitive Staphylococcus aureus (MSSA),
Methicillin resistant Staphylococcus aureus (MRSA), Enterococcus, Klebsiella, E. coli. and $10 \%$ was effective against Acinetobacter.

One of the commonest multi drug resistant (MDR) organism in hospital setting (Acinetobacter) is killed by 10\%, 20\% and $50 \%$ concentration of Manuka Honey.

Similar to few other studies done by Saad B. Almasaudi et al., which showed Manuka honey was effective at 10\%, 20\%, 50\% Concentration against MRSA and MSSA.

Fig.1 Antimicrobial activity of Coorg honey

\begin{tabular}{|l|l|l|l|}
\hline $\begin{array}{l}\text { COORG HONEY } \\
\text { Concentration }\end{array}$ & \multicolumn{1}{|l|}{$10 \%$} & $20 \%$ & $50 \%$ \\
\hline Acinetobater & & \\
\hline CONS & & \\
\hline MRCONS & & \\
\hline MSSA & & \\
\hline MRSA & & \\
\hline Enterococcus & & \\
\hline Klebsiella & & \\
\hline E coli & & \\
\hline ATCC staph & & \\
\hline Pseudomonas & & \\
\hline
\end{tabular}

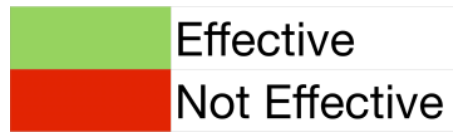

Fig.2 Antimicrobial activity of Kashmir herbal honey
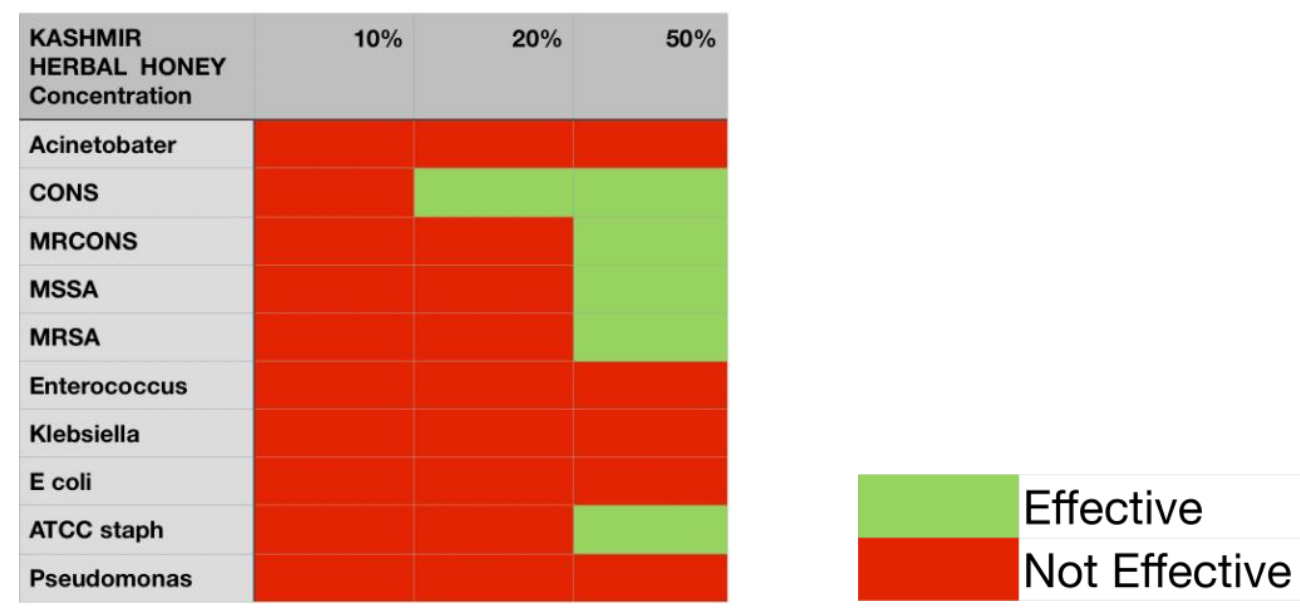
Fig.3 Antimicrobial activity of natural Kashmir honey

\begin{tabular}{|l|l|l|l|}
\hline $\begin{array}{l}\text { NATURAL } \\
\text { KASHMIR HONEY } \\
\text { Concentration }\end{array}$ & & & \\
\hline Acinetobater & & & \\
\hline CONS & & \\
\hline MRCONS & & \\
\hline MSSA & & \\
\hline MRSA & & \\
\hline Enterococcus & & \\
\hline Klebsiella & & \\
\hline E coli & & \\
\hline ATCC staph & & \\
\hline Pseudomonas & & \\
\hline
\end{tabular}

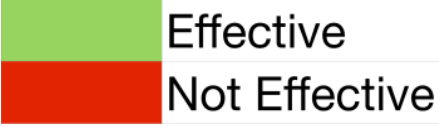

Fig.4 Antimicrobial activity of Dabur honey

\begin{tabular}{|c|c|c|c|}
\hline $\begin{array}{l}\text { DABUR HONEY } \\
\text { Concentration }\end{array}$ & $10 \%$ & $20 \%$ & $50 \%$ \\
\hline Acinetobater & & & \\
\hline CONS & & & \\
\hline MRCONS & & & \\
\hline MSSA & & & \\
\hline MRSA & & & \\
\hline Enterococcus & & & \\
\hline Klebsiella & & & \\
\hline E coli & & & \\
\hline ATCC staph & & & \\
\hline Pseudomonas & & & \\
\hline
\end{tabular}

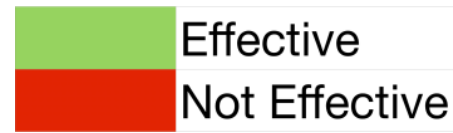

Fig.5 Antimicrobial activity of Manuka Honey

\begin{tabular}{|c|c|c|c|}
\hline MANUKA HONEY & $10 \%$ & $20 \%$ & $50 \%$ \\
\hline \multicolumn{4}{|l|}{ Acinetobater } \\
\hline \multicolumn{4}{|l|}{ CONS } \\
\hline \multicolumn{4}{|l|}{ MRCONS } \\
\hline \multicolumn{4}{|l|}{ MSSA } \\
\hline \multicolumn{4}{|l|}{ MRSA } \\
\hline \multicolumn{4}{|l|}{ Enterococcus } \\
\hline \multicolumn{4}{|l|}{ Klebsiella } \\
\hline \multicolumn{4}{|l|}{ E coli } \\
\hline \multicolumn{4}{|l|}{ ATCC staph } \\
\hline Pseudomonas & & & \\
\hline
\end{tabular}


In our study Manuka honey was found to be effective against 50\% Concentration of Pseudomonas aeruginosa in contrary to few other studies Aled E. L. Roberts et al., which showed Manuka honey is effective against $10 \%, 20 \%, \quad 50 \%$ Concentration of Pseudomonas aerugenosa. This study shows, Manuka. Honey can be used as an topical agent in burns patients.

In our study apart from Manuka honey few other brands of honey (Coorg honey, Kashmir Herbal honey, Natural Kashmir honey and Dabur honey) were also tested at various Concentration which showed all brands of honey have antimicrobial property at $50 \%$ Concentration and it can be used as a topical agent to various infections.

There are so many other studies carried out on antimicrobial effect which concludes that Manuka honey is the best for antimicrobial effect.

In conclusion, though Manuka honey is the best for treating microorganisms, it is not affordable to low socioeconomic people, because of its high cost. The next honey which is effective against organisms is Dabur, and it could be affordable. Though this honey is not as effective as Manuka honey, it is effective against all organisms we used at $50 \%$ concentration. So in our study we would like to conclude honey in general can be used as a topical agent against burns and few other infections caused by commonest microorganisms and honey has little effect in improving the immune status of the individual.

\section{References}

Abd-El Aal, A., El-Hadidy, M., El-Mashad, N., El-Sebaie, A., 2007. Antimicrobial effect of bee honey in comparison to antibiotics on organisms isolated from infected burns. Ann. Burns Fire Disasters 20, 83.

Aiken, A., Karuri, D., Wanyoro, A., Macleod, J., 2012. Interventional studies for preventing surgical site infections in sub-Saharan Africa-a systematic review. Int. J. Surg. 10, 242-249.

Alsarra, I.A., 2009. Chitosan topical gel formulation in the management of burn wounds. Int. J. Biol. Macromol. 45, 1621.

Brady NF, Molan PC, Harfoot CG. The sensitivity of dermatophytes to the antimicrobial activity of manuka honey and other honey. Pharm Sci 1997; 2: 13.

Efem SE. Recent advances in the management of Fournier's gangrene:

French, V., Cooper, R.A., Molan, P.C., 2005. The antibacterial activity of honey against coagulase-negative staphylococci. J. Antimicrob. Chemother. 56, 228-231.

Lu, J., Turnbull, L., Burke, C.M., Liu, M., Carter, D.A., Schlothauer, R.C., Whitchurch, C.B., Harry, E.J., 2014. Manuka-type honeys can eradicate biofilms produced by Staphylococcus aureus strains with different biofilmforming abilities. Peer J 2, e326.

Mandal, M.D., Mandal, S., 2011. Honey: its medicinal property and antibacterial activity. Asian Pac. J. Trop. Biomed. 1, 154-160.

Molan PC.The antibacterial activity of honey. 1.The nature of the antibacterial activity. BeeWorld 1992; 73(1): 5-28.

P.C. Molan. (18 January 2013). Honey as a tropical antibacterial agent for treatment of infected wounds. [Online]. Available: http://

www.worldwidewounds.com/2001/nove mber/Molan/honey-as-topicalagent.html.

Preliminary observations. Surgery, 1993; 113:200-204 
Saleh, I., Barbour, E., Kumosani, T., Harakeh, S, 2011. Cheese as a Reservoir for Antimicrobial Resistance of Escherichia coli and Staphylococcus spp. advances in medicine and biology. Nova Science Publishers, Inc.,
Hauppauge, NY11788.

Theunissen, F., Grobler, S., Gedalia, I., 2001. The antifungal action of three South African honeys on Candida albicans. Apidologie 32, 371-379.

\section{How to cite this article:}

Aswin, P.S. and Neelusree, P. 2019. To Study the Antimicrobial Properties of Honey on Common Microorganisms. Int.J.Curr.Microbiol.App.Sci. 8(09): 114-121.

doi: https://doi.org/10.20546/ijcmas.2019.809.016 